\title{
Performance Evaluation of 802.11 WLAN Scenarios in OPNET Modeler
}

\author{
Kritika Sharma \\ Lecturer \\ Lyallpur Khalsa College \\ Jalandhar
}

\author{
Nitin Bhatia \\ Assistant Professor \\ D.A.V. College \\ Jalandhar
}

\author{
Namarta Kapoor \\ Lecturer \\ D.A.V. College \\ Jalandhar
}

\begin{abstract}
Telecommunications and data networking fields are being changed by the use of wireless networks proving flexibility and mobility of clients as well as servers. This also provides the ability of extension of applications in many diverse areas. In this work, we are evaluating performance of 802.11 WLAN scenarios in Opnet Modeler 14.5. Throughput of the WLAN is evaluated in the presence of high priority traffic as well as low priority traffic, generating data simultaneously. Results are shown in detail with the help of graphs.
\end{abstract}

\section{Keywords}

IEEE802.11 Wireless LAN, Medium Access Control (MAC), Quality-of-Service (QoS), DCF.

\section{INTRODUCTION}

The wireless networks are making integrated networks a reality by bringing fundamental changes to data networking, telecommunication. A wireless network enables people to communicate and access applications and information without wires anywhere and anytime. This provides freedom of movement and the ability to extend applications to different areas. Wireless networking has witnessed an explosion of interest from consumers in recent years due to its applications in mobile and personal communications. This network is getting popular nowadays due to easy to setup feature. One can connect computers without the need for wires. All the communication in the world including satellite communication, mobile communication, internet, telephones and WANs is due to the networking. Wireless networks have changed the era of life. Users are happy to get the data on time and without any problem. The use of the authentication and biometrics and other security mechanisms can improve the security to some extent. Still Hackers' can exploit the sensitive data. The main purpose of the wireless networks is to establish a secure, fast and reliable communicate channel among the people. Wireless network has four types: (a) Wireless Personal Area Networks, (b) Wireless Local Area Networks, (c) Wireless Metropolitan Network, (d) Wireless Wide Area Networks [2]. This paper is most concerned about the study of wireless local area networks.

\subsection{Introduction to Wireless LAN}

Wireless local area network, an emerging and innovative activity in the field of computer networks, supported by flexibility and mobility, in turn attracts the interests of various academia and industry people [4]. A wireless network makes its users capable to connect their mobile systems to the enterprise network instantly with an almost effortless approach. One of the main benefits of wireless is its scope to the distant areas where cabling would be costlier and difficult. Mobility remains the most attractive feature of wireless networks which allows its users to move within the network which in turn also attracts wireless internet service providers' interest in the exploitation of wireless networks. The most influential factors of wireless networks is the provision of higher data rates, lower packet losses and a fair level of Quality of Services. Different types of traffic flows whether it is data flows or multimedia flows like real time voice, streaming voice, video demands access to high data rates and guaranteed QoS in terms of higher throughput, less delay, less no. of collisions and lower packet losses, whereas achieving these factors is very difficult as wired networks is highly time variant and noisy. These requirements led the engineers of IEEE association to keep on working upon the improvement of WLAN standards so that wireless users can satisfy their usage demands from wireless networks. IEEE 802.11 is the most commonly used standard of WLAN [1]. In time, there has been a tremendous growth in the deployment of WLAN standards; network traffic has also been classified as multitude of classes where each class requires a different level of service from the network. Moreover, invention of time bounded applications like VOIP or video streaming requires hard real time constraints. So, it becomes implied that, the WLAN standards should meet these requirements of service differentiation and prioritization. Unfortunately, IEEE802.11 WLAN standard does not satisfy the constraints of QoS parameters. Therefore, IEEE has evolved with an enhanced version IEEE 802.11e which implements QoS mechanisms to a fair level [3]. Many investigations have been made in the performance of 802.11e standard. IEEE 802.11 working group has enhanced the MAC sublayer of the standard to support the QoS constraints. Implementing QoS constraints in MAC layer was very crucial, the main reason being the efficient utilization of the medium by each traffic class keeping priorities in mind.

\section{MAC LAYER PROTOCOLS}

In WLAN scenario, QoS is implemented at MAC layer to achieve access control strategy, addressing, frame check sequencing and security. IEEE 802.11 WLAN standard implemented either as infrastructure mode or ad hoc mode implements a MAC layer functions, $\mathrm{HCF}$ (Hybrid Coordination Function) which combines the mandatory DCF(distributed Coordination function) function and the optional PCF (Point Coordination Function). DCF is contention based whereas PCF is contention free protocol. It has been observed by researchers that, 802.11 MAC function does not meet QoS constraints [9]. In DCF, the stations have to contend the medium before transmission. For this contention mechanism, CSMA/CA algorithm has been employed [6]. Multiple stations can access 
the medium avoiding the collisions in half duplex mode of wireless transmission. According to CSMA/CA, each station that wants to access the medium first senses it using either PHY carrier sensing or Virtual carrier sensing at PHY MAC layer. PHY carrier sensing technique detects packets from other stations wanting to access the medium be sensing the relative signal strength of each station. Whereas, in virtual carrier sensing, a station informs other stations in same basic service set about the reserved duration for its frame transmission. For this, a duration field in the MAC header of MSDU (MAC Service Data Unit) is specified. An inter frame space time is set for each MSDU called DIFS (Distributed Inter Frame Space), which is the time period station has to wait before the station can transmit when the medium is idle. If the medium is free for DIFS time period, the station can transmit, but if the medium is not idle, a binary exponential back_off algorithm gets activated and a back_off counter value is uniformly chosen from the contention window range $[0, \mathrm{CW}]$ using the formula back_off=rand ()$^{*}$ aslot_time. Here, aslot_time is a PHY layer characteristic value. $\operatorname{Rand}()$ is a function that randomly draws an integer from the $\mathrm{CW}$ range $[0, \mathrm{CW}]$, where $C W \min \leq C W \leq C w \max$. $\mathrm{CW}$ is set to 0 at the first transmission. At each retransmission, $\mathrm{CW}$ gets doubled according to $C W=2(C W+1)-1$ and back_off time is rechosen with the increased probability of lesser collisions due to increased back_off time.

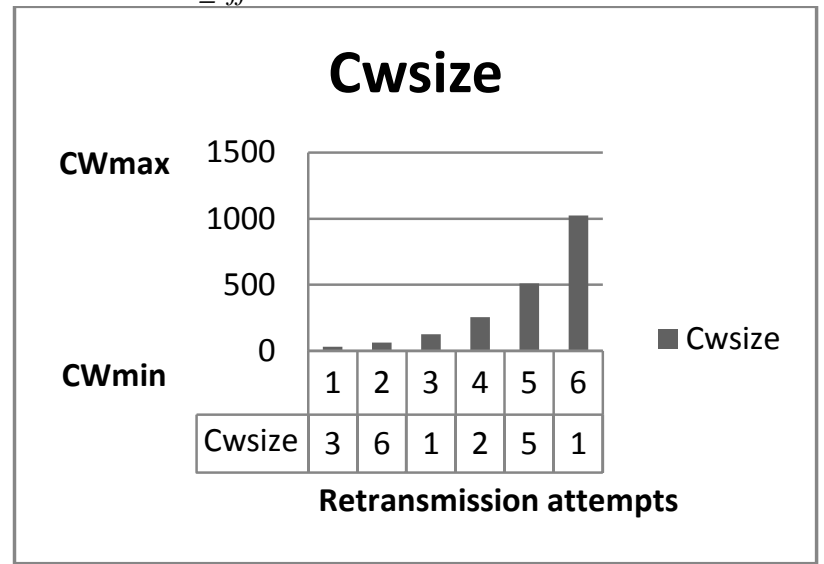

Figure 1: Contention window sizes at each retransmission attempt.

The procedure continues until either $\mathrm{CW}$ reaches Cwmax or at the successful transmission occurs. After reaching Cwmax, CW is set to Cwmin. If during back_off procedure, the medium again gets busy, the back_off counter is freezed, and starts again when the medium gets idle again. After reaching back_off time $=0$, the medium is sensed idle for DIFS, transmission can be made, otherwise retransmission attempt is made.

CA scheme is implemented to reduce the probability of collisions by acknowledgement frames also. Receiving stations send the ACK frame after SIFS time period. Not receiving the ACK frame, the MAC layer at sender retransmits the frame until it reaches its retransmission limit.

However contention window size remains same for all traffic classes [Cwmin-31: Cwmax-1023: DIFS: $50 \mu \mathrm{sec}$ ], thus, it does not support traffic differentiation and cannot generate priorities to real time or delay bounded data [23]. DCF support only best effort services. Services that require higher priority over other types of traffic as VOIP, video do not get fair level of Quality of their services. Another problem with PCF and DCF is that they do not support admission control mechanisms, so the performance of the function degrades when the traffic load is high.

IEEE 802.11e implements enhanced MAC layer functions, which can be classified as station based DCF enhancement or queue based PCF enhancement functions. In 802.11e, HCCA function is implemented that combines Enhanced Distributed Channel Access (EDCA) DCF based enhancement function that operates concurrently with polling based HCF Coordination Channel Access (HCCA) function. The stations and Access Points with QoS mechanism implemented are called QSTA and QAP respectively.802.11e EDCA has been specifically designed to enhance the contention issues of DCF. Each data packet coming from higher layers is assigned a specific user priority value according to the type of the service (voice, video, best effort, background traffic). EDCA maintains FIFO queues at MAC layer which are called Access categories. Each packet is assigned a specific queue or AC according to its priority. Each $\mathrm{AC}$ is implemented as a separate DCF entity having its own prioritization parameters Cwmin and Cwmax, AIFS [AC], and $\mathrm{TXOP}_{\text {limit }}[\mathrm{AC}][5]$. These parameters are generated by QAP in periodic beacon frames. The values of these parameters decide the medium access by each AC according to table 1 .

Table 1: Parameter Set for EDCA

\begin{tabular}{|c|c|c|c|c|c|}
\hline $\mathrm{AC}$ & UP & $\begin{array}{l}\text { Service } \\
\text { Supported }\end{array}$ & $\begin{array}{l}\mathrm{CW} \\
\mathrm{min}\end{array}$ & $\begin{array}{l}\text { CW } \\
\max \end{array}$ & AIFS \\
\hline \multirow[t]{2}{*}{0} & 1 & Background & \multirow[t]{2}{*}{31} & \multirow[t]{2}{*}{1023} & \multirow[t]{2}{*}{ 7*Slot_Time+SIFS } \\
\hline & 2 & Standard & & & \\
\hline \multirow[t]{2}{*}{1} & 0 & Best Effort & \multirow[t]{2}{*}{31} & \multirow[t]{2}{*}{1023} & \multirow[t]{2}{*}{$4 *$ Slot_Time+SIFS } \\
\hline & 3 & $\begin{array}{l}\text { Excellent } \\
\text { Effort }\end{array}$ & & & \\
\hline \multirow[t]{2}{*}{2} & 4 & $\begin{array}{l}\text { Streaming } \\
\text { Multimedia }\end{array}$ & \multirow[t]{2}{*}{15} & \multirow[t]{2}{*}{31} & \multirow[t]{2}{*}{$2 *$ Slot_Time+SIFS } \\
\hline & 5 & $\begin{array}{l}\text { Interactive } \\
\text { Multimedia }\end{array}$ & & & \\
\hline \multirow[t]{2}{*}{3} & 6 & Voice & \multirow[t]{2}{*}{7} & \multirow[t]{2}{*}{15} & \multirow[t]{2}{*}{$2 *$ Slot_Time+SIFS } \\
\hline & 7 & Reserved & & & \\
\hline
\end{tabular}

The smaller the value of theses parameters, the higher is the priority of the $\mathrm{AC}$, and thus shorter will be the delay. When EDCA is active in contention period, each $\mathrm{AC}$ within a station is assigned a TXOP value, which decides the duration for which an AC can contend the medium. Before starting the transmission, the medium is sensed for AIFS (Arbitration Inter Frame Space). AIFS is the time period the station has to wait before accessing the channel, that is, the backoff procedure for the particulate AC is decremented. EDCA uses different values for $\mathrm{CW}$ range for each AC, Cwmin and Cwmax. Smaller values of Cwmin allows high priority traffic to access the medium before lower priority traffic as the backoff time is will be chosen from lower range of values, backoff time will decrement sooner allowing the AC to contend the medium.

\section{RELATED WORK}

Many researches have been done on the performance analysis of the MAC protocols of IEEE standards, most of them concentrating on the improvement of QoS parameters of wireless networks $[8,9,10,12]$. For instance, G. Bianchi et al. [8], analyzed prioritization functions EDCA and DCF with differentiating contention window parameters and AIFS 
parameters against metrics like throughput, delay and also detailed level metric like per slot occupancy probability and he concluded that AIFS differentiation is better than CWmin differentiation. Q. Ni [9] studied the QOS enabled 802.11e standard against the limitations of legacy 802.11and evaluated the performance of EDCA with respect to mean delays. He also evaluated HCCA functions and proposed adaptive tuning mechanism for EDCA and adaptive scheduling algorithm for HCCA. M. Sarkar et al. [10] proposed new QoS support schemes that can differentiate high priority traffic over low priority traffic and also compared it against 802.11e QoS enabled function, EDCA. It is evaluated that the performance of the background traffic is degraded because of multimedia traffic the effect of multimedia traffic on background traffic also considering packet reception power threshold of the receiver, so a new analytical model is proposed to improve the dependence of QoS parameters on background traffic. M. Ahmed et al. [12] analyzed service differentiation mechanisms and proposed an analytical model that implements multilevel priority schemes to control throughput and delay of different traffic categories.

A. Shklyaeva [7] evaluated that EDCF gives better throughput for multimedia traffic however suffering from higher number of collisions because of lesser size of contention window for voice and video type traffic. J. Sengupta and G.S. Grewal [13] evaluated the performance of DCF and EDCF functions with respect to various access categories each of them supporting different types of traffic also pointing out the issue of degraded quality in lower priority traffic in case of starvation. R. Acharya et al. [14] compared the contention based EDCF function with contention free HCCA function where both implements QoS in 802.11e standard against total data carrying capacity of the both functions.

Work has also been done to improve the performance of DCF function, for instance, B. Anjum and A.Z. Uzmi [11] analyzed the service differentiation mechanisms in DCF and to support service differentiation and increased Quality of Service (QoS), they propose two enhancements to the procedures used for managing contention window in original protocol. They also proposed GDMC, a new scheme for service differentiation in 802.11 WLANs.

Researchers [15, 16] have worked upon proposing new prioritization schemes to improve the service differentiation. A. Thangaraj et al. [15] studied that the performance of TCP traffic is not up to the mark as they are not given priority over UDP traffic and then a new prioritization scheme is proposed for TCP traffic which enhances the overall throughput of the network IEEE 802.11e. J. Lv [16] considered service differentiation not only between different access categories supporting different types of traffic but also within each access category so that overall throughput can be increased. However, S. Mangold et al. [17] focused on the performance study of AP environments with negative-exponentially distributed inter arrival times. A. Lindgren et al. [18] also included the concept of distributed fair scheduling and blackburst in the performance investigation of MAC layer functions of 802.11 and 802.11e and concluded with Black burst as the best choice amongst them. A. Grilo and M. Nunes [19] investigated with a limiting factor in the simulation scenario where each station generating at most only one type of traffic. H.L. Truong and G. Vannuccini [20] tested 802.11e standard in different scenarios where each scenario is testing for different type of traffic class such as (VOIP, video, data). M. EDCA has resulted better in throughput and access delay time in case of heterogeneous traffic types like non-bursty, bursty and self-similar [21]. Kanthali et al. [22] compared EDCA and DCF functions against different basic service set simulations scenarios; (i) wireless networks (ad-hoc networks) (ii) wired cum wireless network (infrastructure networks) against QoS metrics as throughput, delay and packet loss.

In this paper, we study throughput and traffic generation parameters of 802.11 WLAN.

\section{PROPOSED SYSTEM}

In the proposed system, we are evaluating performance of 802.11 WLAN scenarios in Opnet Modeler 14.5. Throughput of the WLAN is evaluated in the presence of high priority traffic as well as low priority traffic, generating data simultaneously. For this evaluation, we configure some wireless stations as clients and some as servers of different traffic flows. 8 stations have been configured to support four types of applications: HTTP, Remote Login, VC (Video Conferencing) and VOIP (Voice over IP). All type of data flows among clients and servers simultaneously.

Table 2. No. of Clients for each type of service

\begin{tabular}{|l|l|}
\hline \multicolumn{2}{|c|}{ Client stations } \\
\hline Node_2 & Client for HTTP service \\
\hline Node_3 & Client for all 4 services \\
\hline Node_4 & Client for all 4 services \\
\hline
\end{tabular}

Table3. No. of Servers for each type of service

\begin{tabular}{|l|l|}
\hline \multicolumn{2}{|l|}{ Server Stations } \\
\hline Node_5 & Server for all services \\
\hline Node_6 & $\begin{array}{l}\text { Server for Video Conferencing and } \\
\text { VOIP }\end{array}$ \\
\hline Node_7 & Server for Remote_Login \\
\hline Node_8 & Server for all services \\
\hline Node_17 & Server for HTTP and Remote_Login \\
\hline
\end{tabular}

We can also observe that number of clients for low priority traffic HTTP and Remote Login traffic are three, whereas number of clients for high priority traffic VC and VOIP are two. PHY characteristics of our proposed WLAN scenario is based upon 802.11b PHY characteristics supporting Data rate of 11 mbps.

\subsection{Traffic Sent By All Services}

In the proposed system we will study traffic sent over WLAN by each type of traffic and throughput achieved by WLAN over 1 hour time.

\subsubsection{Traffic Sent By HTTP}

In this scenario, number of clients and servers of HTTP traffic are three. Global statistic of HTTP Traffic sent for Packets/sec is recorded.

We can observe that HTTP traffic is generated and is soon degraded in the presence of other high priority traffic. 


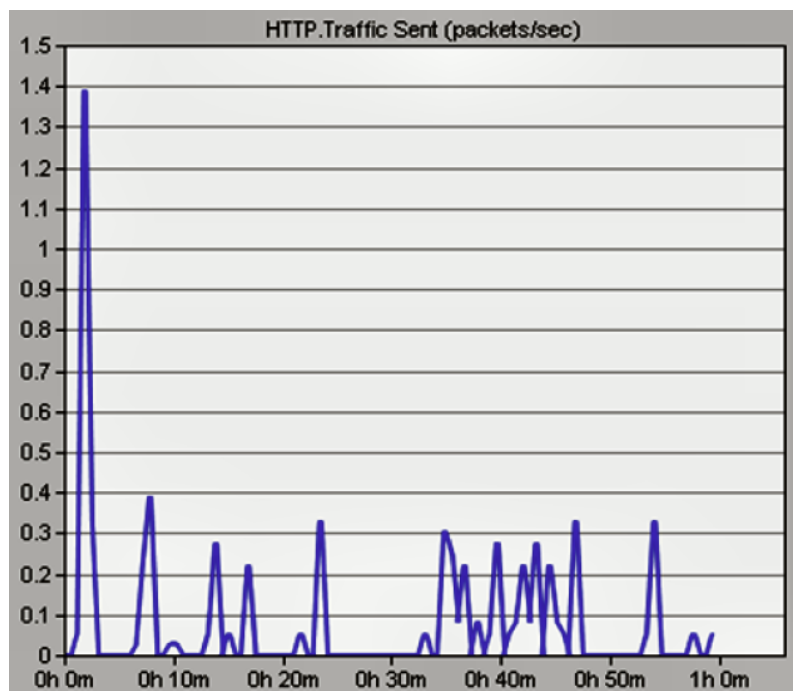

Figure 2: Traffic sent by HTTP

\subsubsection{Traffic Sent By Remote Login}

In the proposed scenario, number of clients and servers for Remote_Login are three. Traffic (packets/sec) sent over network is gathered and studied.

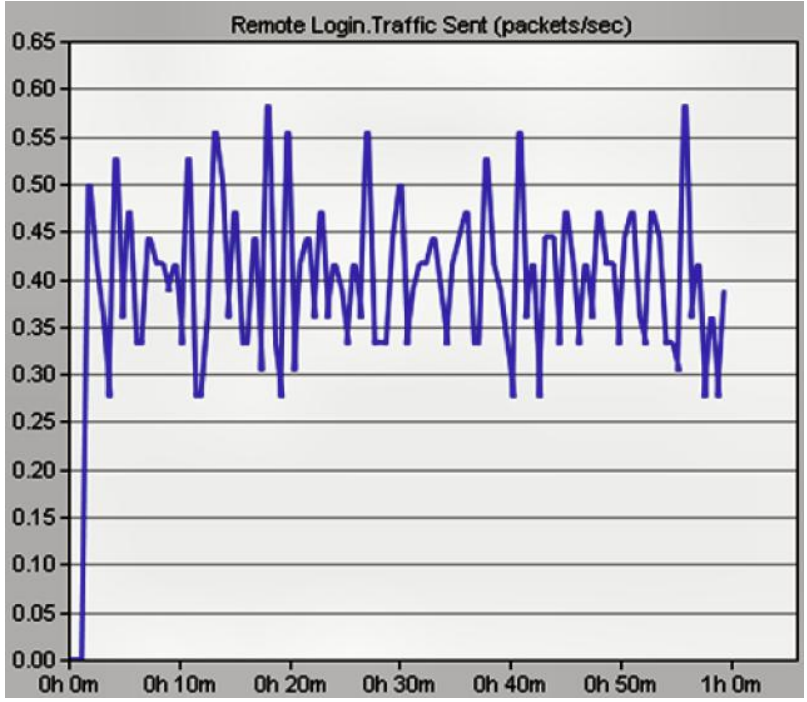

Figure 3: Traffic sent by Remote Login

Here, we can observe that Remote_Login service is being considered of higher priority than HTTP. Traffic sent by Remote_Login is at fair level but having fluctuations in the presence of other high priority traffic.

\subsubsection{Traffic sent by Voice Conferencing}

Considered as delay sensitive traffic, VC gets priority over the network. Number of clients and servers supporting Video Conferencing service are two. Traffic sent is much higher of this service than in other types of services as in figure 4 .

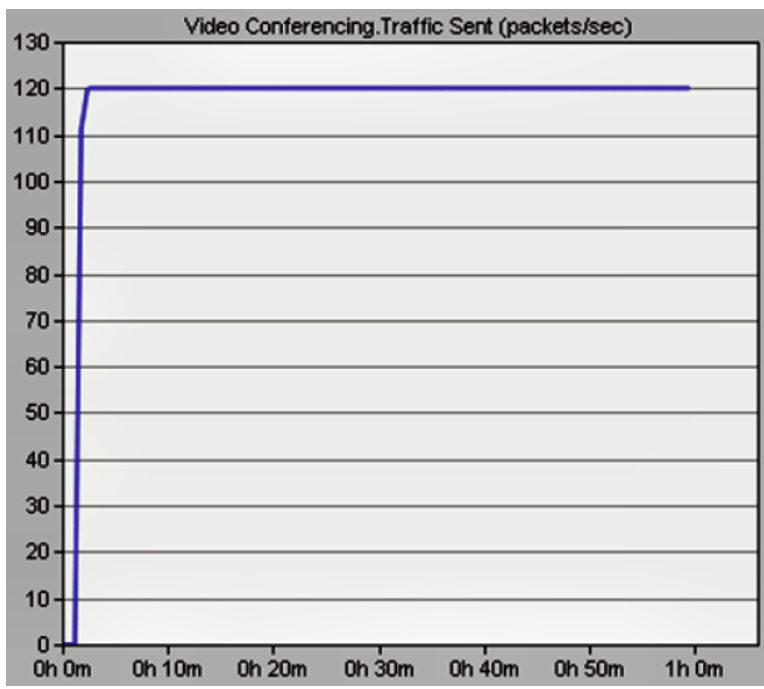

Figure 4: Traffic Sent by Video Conferencing

After a minute of time, the traffic is being sent constantly over the network without any interference by other traffic flows.

\subsubsection{Traffic Sent by Voice}

Voice traffic is also considered as delay sensitive and gets priority over the network. Number of clients and servers supporting Voice type of service are two. Traffic sent over network is shown in figure 5 .

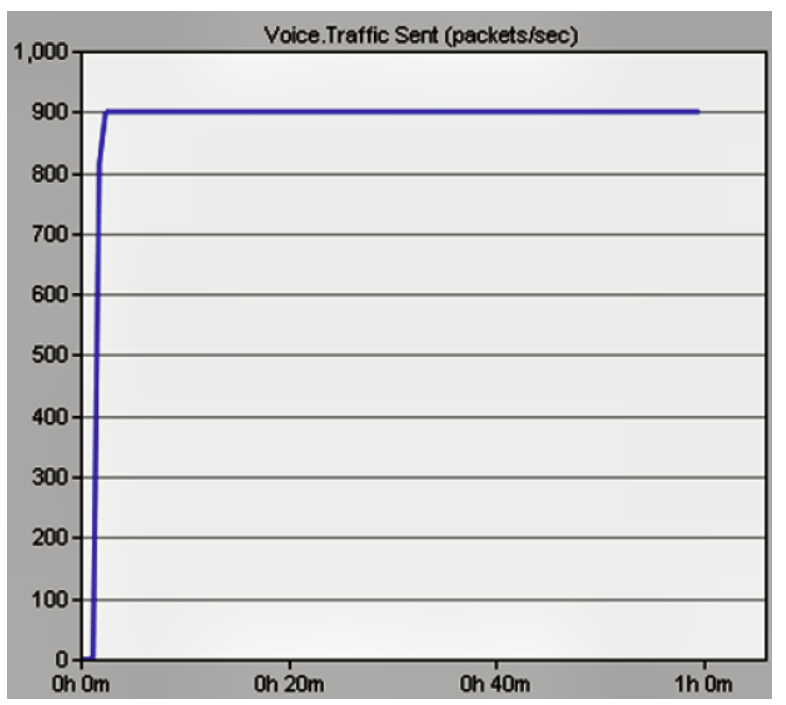

Figure 5: Traffic Sent by Voice

Here also, the traffic is generated in a great amount sending almost 900 packets $/ \mathrm{sec}$, and after some time the traffic flow becomes constant. Here we can observe that the performance of Voice traffic is better that $\mathrm{VC}$, as its throughput is much higher than of VC.

\subsection{Throughput of 802.11 Wireless LAN}

In this WLAN scenario, global statistic of WLAN throughput is gathered for 1 hour of simulation time. We can observe that as soon as the simulation begins and the traffic starts flowing, the throughput of WLAN rises with huge amount and becomes 
almost constant with little fluctuations caused by noise and interferences. This is achieved through constant traffic generation by high priority traffic

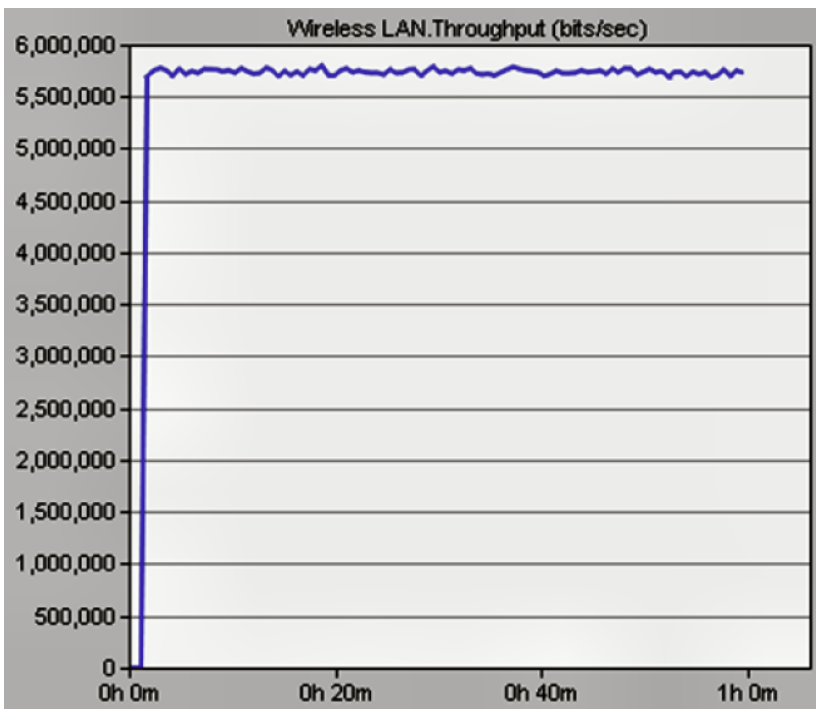

Figure 6: Throughput achieved by WLAN

\section{CONCLUSION}

In this paper, MAC layer protocols for 802.11/e has been studied, responsible for QoS achievement in WLAN. The performance of 802.11 based WLAN has been analyzed using simulation based study. It has been observed that though no. of nodes generating low priority traffic (HTTP, R_Login) is higher, the data flow of these services is quite low as compared to high priority traffic with lesser no. of supporting nodes. This prioritization is achieved at the cost of degradation in best effort services which are also considered to be supported by various users. It has also been observed that throughput of WLAN becomes almost constant after some time which also effects the performance of network.

\section{REFERENCES}

[1] P802.11 , Draft Standard for wireless LAN medium access control (MAC) and physical layer (PHY) specification, IEEE (1997) .

[2] Chelouche, M., Hethuin, S. and Ramel, L. 1997. Digital wireless broadband corporate and private network: RENT concepts and applications. IEEE Commun. Mag. (Jan. 1997) 42-51.

[3] IEEE 802.11 WG. Draft Supplement to Standard for Telecommunications and Information Exchange between Systems-LAN/MAN Specific Requirements - Part 11: Wireless MAC and PHY Specifications: MAC Enhancements for QoS. IEEE 802.11e/draft 11.0. (Oct. 2004).

[4] Pilat, P. 2000. Home RF-SWAP: Optimized for home networking, Intel Developer Update Mag. (Jun. 2000) 1-5.
[5] Wireless LAN Medium Access Control (MAC) and Physical Layer (PHY) Specification, Tech. Rep. 802.11. (1999) IEEE Network.

[6] Eustathia, Z. and Theodore A.2002. CSMA/CA performance under high traffic conditions: throughput and delay analysis. Computer Communications. (2002)313-321.

[7] Shklyaeva, A., Kubanek, D. and Novotny, V. 2007. Analysis of IEEE 802.11e for Delay Sensitive Traffic in Wireless LANs. Proceedings of the Sixth International Conference on Networking. (Aug. 2007).

[8] Bianchi, G., Tinnirello, I. and Scalia, L. 2005. Understanding 802.11e contention-based prioritization mechanisms and their coexistence with legacy 802.11 stations. IEEE Network. (July-Aug. 2005) 28-34.

[9] Ni, Q. 2005. Performance analysis and enhancements for IEEE 802.11e wireless networks. IEEE Network. (JulyAug. 2005) 21-27.

[10] Sarkar, M., Ray, M. and Nuggehalli, P. 2008. Evaluating a QoS-supportive MAC layer protocol for WLANs. Proceedings of IEEE Military Communications Conference. (May 2008) 1-7.

[11] Anjum, B. and Uzmi, Z. A. 2007. Multi-Class QoS in 802.11 Networks using Gentle Decrease of Multiple Contention Windows. IEEE Network. (2007) 5242-5247.

[12] Ahmed, M., Fadeel, G.A. and Ibrahim, I. 2010. Differentiation between Different Traffic Categories Using Multi-level of Priority in DCF-WLAN. Sixth Advanced International Conference on Telecommunications. (2010) 263-268

[13] Gupta, J. S. and Grewal, G.S. 2010. Performance evaluation of IEEE 802.11 MAC layer in supporting delay sensitive services. IJWMN. (February 2010) 42-53.

[14] Acharya, R., Vityanathan, V. and Chellaih, P.R. 2010. WLAN QoS Issues and IEEE 802.11e QoS Enhancement, International Journal of Computer Theory and Engineering. (February 2010) 1793-8201.

[15] Thangaraj, A., Zeng, Q.A. and Li, X. 2008. Performance Analysis Of The IEEE 802.11e Wireless Networks With TCP ACK Prioritization. IEEE Network. (2008) 1-6.

[16] Lv, J., Zhang, X. and Han, X. 2008. A Novel Dynamic Tuning of the Contention Window (CW) for IEEE 802.11e Enhanced Distributed Control Function. Fourth International Conference on Networked Computing and Advanced Information Management. (2008) 62-67.

[17] Mangold, S., Choi, S., Hiertz, G., Klein, O. and Walke, B. 2003. Analysis of IEEE 802.11e for QoS support in wireless LANs. IEEE Wireless Communications. (2003) $40-50$.

[18] Lindgren, A., Almquist, A. and Schelen, O. 2001. Evaluation of quality of service schemes for IEEE 802.11 wireless LANs. 26th Annual IEEE Conference on Local Computer Networks. (2001) $348-351$. 
[19] Grilo, A. and Nunes, M. 2002. Performance evaluation of IEEE 802.11e. Proc. 13th IEEE International Symposium, Personal, Indoor and Mobile Radio Communications. (2002). 511-517.

[20] Truong, H.L. and Vannuccini, G. 2003. Performance evaluation of the QoS enhanced IEEE 802.11e MAC layer. Proc. Of $57^{\text {th }}$ IEEE Semi-annual, Vehicular Technology Conference. (2003). 940-944.

[21] Mamun, I., Tair A. and Min, G. 2006. Performance Evaluation of an Enhanced distributed Channel Access
Protocol under Heterogeneous Traffic. IEEE Network. (2006).

[22] Kanthali, M., Gaiwak, A. and Vyavahare, P.D. 2007. Performance comparison of contention-based channel access mechanism of IEEE 802.11 and 802.11e. 7th IEEE International Conference on Computer and Information Technology. (2007) 503-510.

[23] Malik, S. A., Abbas, A. and Shah, M.A. 2010. QoS analysis of IEEE 802.11/IEEE 802.11e MAC Protocols. AJBAS, INSInet Publication. (2010) 1439-1448. 